\title{
NOETHERIAN DOMAINS WITH MANY MORE ELEMENTS THAN HEIGHT-ONE PRIMES
}

\author{
D. D. ANDERSON
}

(Communicated by Wolmer Vasconcelos)

\begin{abstract}
Extending previous results of L. Claborn and H.W. Lenstra, Jr., we show that if $D$ is a Krull domain with a set of height-one primes $X^{(1)}$ that satisfies either (1) $D$ contains a subset $k$ with $|k|>\left|X^{(1)}\right|$ and for $\mu \neq \lambda \in k$, $\mu-\lambda$ is a unit, or (2) $|D|>\left|X^{(1)}\right|^{\wedge_{0}}$, then $D$ is a Euclidean domain. We also show that any Noetherian ring satisfying (1) or Noetherian domain satisfying (2) has Krull dimension at most one.
\end{abstract}

It is a well-known consequence of the Principal Ideal Theorem that a Noetherian domain (or ring) with only a finite number of height-one primes has Krull dimension at most one. (For example, see the proof of [5, Theorem 144]. It is also well known that a Dedekind domain (even a Krull domain) with a finite number of height-one primes is a semilocal PID. Moreover, Estes and Ohm [3, Corollary 5.4] and Samuel [7, Proposition 5] showed that a semilocal PID is a Euclidean domain with (smallest) algorithm $\phi(x)=\sum_{P \in X^{(1)}} v_{P}(x)$. Here for any ring $R, X^{(1)}=X^{(1)}(R)$ is the set of height-one prime ideals of $R$, and for a Krull domain $D$ and $P \in X^{(1)}(D), v_{P}$ is the normalized discrete valuation associated with $P$. And we define an integral domain $D$ to be a Euclidean domain if there exists an ordinal number $W$ and a function $\phi: D-\{0\} \rightarrow W$ satisfying the condition: for $a, b \in D$ with $b \neq 0$, there exist $q, r \in D$ with $a=b q+r$ and either $r=0$ or $\phi(r)<\phi(b)$. Then $\phi$ is called the algorithm for $D$. Claborn [2] (or see [4, Corollary 13.10]) "generalized" this result concerning Dedekind domains by showing that if $D$ is a Dedekind domain with $|D|>\left|X^{(1)}\right|^{\aleph_{0}}$, then $D$ is a PID. (However, Claborn remarks that a Dedekind domain $D$ with $|D|>\left|X^{(1)}\right|$ need not be a PID. For an example of such a Dedekind domain, see [4, Example 15.16].) Then Lenstra [6] showed that such a Dedekind domain is actually a Euclidean domain with algorithm $\phi: D-\{0\} \rightarrow \omega^{2}$ where $\phi(x)=\omega \cdot \sum_{P \in Z} v_{P}(x)+\sum_{P \in X^{(1)}-Z} v_{P}(x)$ for some finite subset $Z \subseteq X^{(1)}$. We show that Lenstra's result (and hence Claborn's result) holds for an arbitrary Krull domain. We also show that for any Noetherian domain $D$ with $|D|>\left|X^{(1)}\right|^{\aleph_{0}}, \operatorname{dim} D \leq 1$. (We are allowing the possibility that $D$ is a field.)

Received by the editors December 20,1993 and, in revised form, February 16, 1994 and March 12, 1994.

1991 Mathematics Subject Classification. Primary 13E05, 13 F07.

(C) 1995 American Mathematical Society 
But first we generalize another result of Claborn and Lenstra. Claborn [2] (or see [4, Proposition 13.7]) proved that if a Dedekind domain $D$ contains a subfield $k$ with $|D|=|k|>\left|X^{(1)}\right|$, then $D$ is a PID. Lenstra [6] sharpened Claborn's result by assuming only that $k$ is a subset of the Dedekind domain $D$ with $|k|>\left|X^{(\mathbf{1})}\right|$ and $\lambda-\mu \in U(D) \cup\{0\} \quad(U(D)$ is the group of units of $D$ ) for all $\lambda, \mu \in k$ and then concluding that $D$ is a Euclidean domain with algorithm $\phi(x)=\sum_{P \in X^{(1)}} v_{P}(x)$. Our first theorem extends this to arbitrary Krull domains.

Lemma 1. Let $D$ be a Krull domain. Then the cardinality of the set of principal ideals of $D$ generated by irreducible elements is $\left|X^{(1)}\right|$. If $X^{(1)}$ is infinite, the cardinality of the set of principal ideals of $D$ is also equal to $\left|X^{(1)}\right|$.

Proof. Let $I r r$ be the set of principal ideals of $D$ generated by irreducible elements and let $P(D)_{+}$be the set of nonzero (integral) principal ideals of $D$. First, suppose that $X^{(1)}$ is finite. Then $D$ is a semilocal PID with $X^{(1)}=I r r$. Next, suppose that $X^{(1)}$ is infinite. Since each $P \in X^{(1)}$ contains an element of $\mathrm{Irr}$ and each element of $\mathrm{Irr}$ is contained in only finitely many height-one primes, $\left|X^{(1)}\right| \leq|\operatorname{Irr}|$. So $\left|X^{(1)}\right| \leq|\operatorname{Irr}| \leq\left|P(D)_{+}\right|$. Now two nonzero principal ideals $(a)$ and $(b)$ of $D$ are equal if and only if $v_{P}(a)=v_{P}(b)$ for each $P \in X^{(1)}$. Since the set of functions from $X^{(1)}$ to $N$ which are zero almost everywhere has cardinality $\left|X^{(1)}\right|$, we have $\left|P(D)_{+}\right| \leq\left|X^{(1)}\right|$.

Lemma 1 may also be interpreted in terms of the multiplicative group $P(D)$ of nonzero principal fractional ideals of $D$ (called the group of divisibility of $D$ ). Since $P(D)$ is isomorphic to a subgroup of the free abelian group of all functions from $X^{(1)}$ to $\mathbb{Z}$ which are zero almost everywhere, $P(D)$ is itself free. By Lemma 1, $\operatorname{rank} P(D)=\left|X^{(1)}\right|$ and if $X^{(1)}$ is infinite, $\operatorname{rank} P(D)=$ $|P(D)|=|I r r|$.

Theorem 2. Let $D$ be a Krull domain that contains a subset $k$ (e.g., a subfield $k$ ) with $|k|>\left|X^{(1)}\right|$ and $\lambda-\mu \in U(D) \cup\{0\}$ for all $\lambda, \mu \in k$. Then $D$ is Euclidean with smallest algorithm $\phi(x)=\sum_{P \in X^{(1)}} v_{P}(x)$.

Proof. As previously mentioned, the case where $X^{(1)}$ is finite has already been handled in [3] and [7]. So suppose that $X^{(1)}$ is infinite. Let $a, b \in D$ with $b \neq 0$ and $a \notin(b)$. By Lemma 1 , there exist $\lambda \neq \mu \in k$ with $(a+\lambda b)=(a+\mu b)$. Then $(a+\lambda b)=(a, b)$, so $(a+\lambda b)=(a, b)_{v}$, where for a nonzero ideal $I$, $I_{v}=\left(I^{-1}\right)^{-1}$. Then $v_{P}(a+\lambda b)=\min \left\{v_{P}(a), v_{P}(b)\right\} \leq v_{P}(b)$ for all $P \in X^{(1)}$ with strict inequality for at least one $P$. Hence the element $r=a+\lambda b$ has $\phi(r)<\phi(b)$ and $a=b(-\lambda)+r$. Thus $\phi$ is an algorithm. By [7, Corollary 2] $\phi$ is the smallest algorithm.

Corollary 3. Let $R$ be a Noetherian ring that contains a subset $k$ (e.g., a subfield) with the properties that $|k|>\left|X^{(1)}\right|$ and $\lambda-\mu \in U(R) \cup\{0\}$ for all $\lambda, \mu \in k$. Then for each minimal prime ideal $P$ of $R$, the integral closure $\overline{R / P}$ of $R / P$ is a Euclidean domain. Hence $\operatorname{dim} R \leq 1$.

Proof. Let $P$ be a minimal prime ideal of $R$. Then $R / P$ is a Noetherian domain with $X^{(1)}(R / P) \leq X^{(1)}(R)<|k|=|\bar{k}|$ where $\bar{k}=\{\mu+P \mid \mu \in k\}$. Thus $R / P$ satisfies the hypothesis of the corollary, so we may assume that $R$ is a Noetherian integral domain. If $\left|X^{(1)}(R)\right|<\infty$, then $\left|X^{(1)}(\bar{R})\right|<\infty$ and hence $\bar{R}$ is a semilocal PID and thus a Euclidean domain with $\operatorname{dim} R=\operatorname{dim} \bar{R} \leq 1$. 
Suppose that $X^{(1)}(R)$ is infinite. Then $|k|>\left|X^{(1)}(R)\right|=\left|X^{(1)}(\bar{R})\right|$ and $\bar{R}$ is a Krull domain (the equality and the fact that $\bar{R}$ is a Krull domain is part of the Mori-Nagata Integral Closure Theorem [4, Theorem 4.3]). By Theorem 2, $\bar{R}$ is a Euclidean domain. Hence $\operatorname{dim} R=\operatorname{dim} \bar{R} \leq 1$.

Example 4. Note that for any pair of cardinal numbers $\alpha \leq \beta$ with $\beta$ infinite, there is a Euclidean domain $D$ with subfield $k$ satisfying $|k|=|D|=\beta$ and $\left|X^{(1)}(D)\right|=\alpha$. For let $k$ be a field with $|k|=\beta$ and $\left\{f_{j}\right\}_{j \in \Delta}$ a set of nonassociate irreducible polynomials of $k[X]$ with $|\Delta|=\alpha$. Then $D=k[X]_{S}$ where $S=k[X]-\bigcup\left(f_{j}\right)$ has the desired properties. If $\alpha<\beta$ we have seen that $\phi(x)=\sum v_{P}(x)$ is an algorithm for $D$. However, if $\alpha=\beta, \phi$ may or may not be an algorithm for $D$. The smallest algorithm for $k[X]$ is $\theta(f)=\operatorname{deg} f[7$, page 290] and we always have $\phi(f) \leq \theta(f)$, so $\phi$ is an algorithm if and only if every irreducible polynomial of $k[X]$ has degree one, that is, $k$ is algebraically closed. An example where the set $k$ in Theorem 1 is not a field is as follows.

Let $\left\{X_{\lambda} \mid \lambda \in \Lambda\right\}$ be a set of indeterminates over $\mathbb{Z}$ and let $D_{\Lambda}=\mathbb{Z}\left(\left\{X_{\lambda}\right\}\right)=$ $\mathbb{Z}\left[\left\{X_{\lambda}\right\}\right]_{S}$ where $S=\left\{f \in \mathbb{Z}\left[\left\{X_{\lambda}\right\}\right] \mid\right.$ the coefficients of $f$ generate $\left.\mathbb{Z}\right\}$. Then $D_{\Lambda}$ is a Euclidean domain with $X^{(1)}\left(D_{\Lambda}\right)=\operatorname{Spec}\left(D_{\Lambda}\right)-\{0\}=\left\{p D_{\Lambda} \mid p\right.$ is a nonzero prime of $\mathbb{Z}\}$ (see, for example [3]). Take $k=\left\{X_{\lambda} \mid \lambda \in \Lambda\right\}$, so $|k|=|\Lambda|$ and for $X_{\lambda}, X_{\mu} \in k, X_{\lambda}-X_{\mu} \in U\left(D_{\Lambda}\right) \cup\{0\}$. Certainly $|k|$ may be made arbitrarily large.

Lemma 5. Let $D$ be an integral domain containing a prime ideal $Q$ with ht $Q>$ 1 satisfying the following condition: if $0 \neq a \in Q$, then there are only finitely prime ideals $P \subseteq Q$ that are minimal over $(a)$ and any such prime ideal $P$ has height one. Let $X^{(1)}(Q)=\left\{P \in X^{(1)}(D) \mid P \subseteq Q\right\}$. Then $|D| \leq\left|X^{(1)}(Q)\right|^{\aleph_{0}}$.

Proof. Let $b_{1}, b_{2}$ be distinct nonzero elements of $Q$. Let $Q_{1}, \ldots, Q_{S}$ be the elements of $X^{(1)}(Q)$ containing $b_{2}-b_{1}$. Since each $Q / Q_{i}$ is infinite, by [1, Chapter 2, $\S 1$, exercise 1], there exists $b_{3} \in Q-\bigcup_{i=1}^{S}\left(b_{2}+Q_{i}\right)$. So $b_{3}-b_{2}$ is not contained in any of the height-one primes contained in $Q$ that contain $b_{2}-b_{1}$. In a similar manner, we inductively choose an element $b_{n} \in Q \quad(n>3)$ such that for $i<j<n, b_{n}-b_{j}$ is not contained in any element of $X^{(1)}(Q)$ containing $b_{j}-b_{i}$. Let $a \in Q$. For each $n \geq 1$, choose $P_{n} \in X^{(1)}(Q)$ with $a-b_{n} \in P_{n}$. Note that $\left\{P_{n}\right\}$ is infinite. For if $\left\{P_{n}\right\}$ is finite, there exists $i<j<k$ with $P_{i}=P_{j}=P_{k}$. But then $b_{k}-b_{j}, b_{j}-b_{i} \in P_{k}$, a contradiction. Define the function $S: Q \rightarrow\left(X^{(1)}(Q)\right)^{\aleph_{0}}$ by $S(a)=\left(P_{n}\right)$. Now $S$ is injective. For if $a, b \in Q$ with $S(a)=S(b)$, then $a-b=\left(a-b_{n}\right)-\left(b-b_{n}\right) \in P_{n}$ for each $n \geq 1$. Since a nonzero element of $Q$ is contained in only finitely many height-one primes, we must have $a=b$. Thus $|Q| \leq\left|X^{(1)}(Q)\right|^{\aleph_{0}}$. Since $|D|=|Q|$, the result follows.

Theorem 6. Let $D$ be an integral domain satisfying the following two conditions for each maximal ideal $M$ of $D$ : (i) every prime ideal $P$ of $D_{M}$ minimal over a nonzero proper principal ideal of $D_{M}$ has ht $P=1$ and (ii) every nonzero proper principal ideal of $D_{M}$ is contained in only finitely many height-one prime ideals of $D_{M}$. (For example, these two conditions hold if $D_{M}$ is a Krull domain or Noetherian domain for each maximal ideal $M$ of $D$.) Then $\left|X^{(1)}(D)\right|^{N_{0}}<|D|$ implies $\operatorname{dim} D \leq 1$.

Proof. Suppose that $\operatorname{dim} D>1$; so $D$ has a maximal ideal $Q$ with ht $Q>1$. Note that $Q$ satisfies the hypothesis for the prime ideal $Q$ in Lemma 5. Hence 
by Lemma $5,|D| \leq\left|X^{(1)}(Q)\right|^{\aleph_{0}} \leq\left|X^{(1)}(D)\right|^{\aleph_{0}}$, a contradiction. Thus $\operatorname{dim} D \leq$ 1 .

Theorem 7. Let $D$ be a Krull domain with $|D|>\left|X^{(1)}\right|^{\aleph_{0}}$. Then $D$ is a Euclidean domain with algorithm $\phi: D-\{0\} \rightarrow \omega^{2}$ given by $\phi(x)=\omega$. $\sum_{P \in Z} v_{P}(x)+\sum_{P \in X^{(1)}-Z} v_{P}(x)$ for some finite subset $Z \subseteq X^{(1)}$. Hence $\operatorname{dim} D \leq$

Proof. By Theorem 6, $\operatorname{dim} D \leq 1$ and hence $D$ is a Dedekind domain. The result now follows from the previously mentioned result of Lenstra [6].

Corollary 8. Let $D$ be a Noetherian domain with $|D|>\left|X^{(1)}\right|^{\aleph_{0}}$. Then the integral closure $\bar{D}$ of $D$ is a Euclidean domain and hence $\operatorname{dim} D \leq 1$.

Proof. If $\left|X^{(1)}(D)\right|<\infty$, then $D$ is a one-dimensional semilocal domain and hence $\bar{D}$ is a semilocal PID and hence a Euclidean domain. So we may assume that $X^{(1)}(D)$ is infinite. As remarked in Corollary 3, $\bar{D}$ is a Krull domain with $|\bar{D}|=|D|>\left|X^{(1)}(D)\right|^{\aleph_{0}}=\left|X^{(1)}(\bar{D})\right|^{\aleph_{0}}$. Thus $\bar{D}$ is a Euclidean domain and hence $\operatorname{dim} D=\operatorname{dim} \bar{D} \leq 1$.

Corollary 8, unlike Corollary 3 , cannot be extended to Noetherian rings with zero divisors. Let $K$ be a field with $|K|>\aleph_{0}^{\aleph_{0}}$. Then $R=K \times \mathbb{Q}\left[X_{1}, \ldots, X_{n}\right]$ is an $n$-dimensional integrally closed Noetherian ring with $|R|=|K|>\aleph_{0}^{\aleph_{0}}=$ $\left|X^{(1)}(R)\right|^{\aleph_{0}}$. Here $R$ has two minimal prime ideals. However, Corollary 8 does carry over to Noetherian rings with a unique minimal prime ideal.

The author would like to thank the referee whose suggestions greatly improved this paper. In particular, the referee suggested Lemma 1 and Lemma 5 (which is based on the proof in [2]) which lead to simplifications of the original proofs of Theorem 2 and Theorem 7, respectively. The referee also raised the interesting question of whether the assumptions of Theorem 7 imply the existence of a set $k$ as in Theorem 2 .

\section{REFERENCES}

1. N. Bourbaki, Commutative algebra, Hermann, Paris, and Addison-Wesley, Reading, MA, 1972.

2. L. Claborn, A generalized approximation theorem for Dedekind domains, Proc. Amer. Math. Soc. 18 (1967), 378-380.

3. D. Estes and J. Ohm, Stable range in commutative rings, J. Algebra 7 (1967), 343-362.

4. R. Fossum, The divisor class group of a Krull domain, Springer-Verlag, New York, Heidelberg, and Berlin, 1973.

5. I. Kaplansky, Commutative rings, rev. ed., Univ. of Chicago Press, Chicago, 1974.

6. H.W. Lenstra, Jr., Euclid's algorithm in large Dedekind domains, J. Indian Math. Soc. 50 (1986), 41-47.

7. P. Samuel, About Euclidean rings, J. Algebra 19 (i971), 282-301.

Department of Mathematics, The University of Iowa, Iowa City, Iowa 52242

E-mail address: dan-andersonQuiowa.edu 